Result(s)* BAX protein was significantly higher in Type 1 $(p=0.004)$ and Type $2(p=0.024)$ EC compared to atrophic samples; both types showing higher levels in the glandular tissue; only Type 1 EC demonstrated significantly higher stromal staining. By contrast, Bcl-2 expression was significantly $(p=0.017)$ lower only in Type 2 EC. Increased proliferation (Ki-67 staining) of both Type $1(\mathrm{p}=0.003)$ and Type 2 $(\mathrm{p}<0.0001)$ EC was observed. The BAX:Bcl-2 ratio was only significantly $(\mathrm{p}<0.001)$ higher in the Type 2 EC because of glandular expression. BAX expression $(\mathrm{r}=0.615 ; \mathrm{p}=0.0001)$, and the BAX:Bcl-2 ratio $(r=0.507 ; \mathrm{p}=0.003)$ were directly related to proliferation whilst $\mathrm{Bcl}-2$ expression was inversely related $(\mathrm{r}=-0.544 ; \mathrm{p}=0.0009)$.

Conclusion* Since BAX (pro-apoptotic signal) was increased in both types of EC, whilst Bcl-2 (anti-apoptotic signal) was decreased, then increased apoptosis is present in EC tissue. The direct correlation between the BAX:Bcl-2 ratio (indicator of increased or decreased apoptosis) and cellular proliferation indicates that as the tumours grow, they also undergo significant apoptosis. Since these tissues have increased production of anandamide4, then it is probable that anandamide is not stimulating cellular proliferation but is attempting to control tissue hyperplasia through increased apoptosis.

\section{OBTURATIVE NERVE COMPLICATION IN LAPAROSCOPIC TREATMENT OF ENDOMETRIAL CANCER}

E Milnerowicz-Nabzdyk*, M Kalus, K Nowak, J Tomiczek-Szwiec, M Mrugała, A MachnickaRusek. SPZOZ Opolskie Centrum Onkologii im. prof. Tadeusza Koszarowskiego, Opole, Poland

\subsection{6/ijgc-2021-ESG0.161}

Introduction/Background* Intraoperative, iatrogenic demage of periferal nerves are rare but serious operative complication. Miss it or incorect repair can get serious consequences for patient. During laparoscopic pelvic lymphadenectomy, specialy when we remove metastases from obturatory fossa, the risk of demage obturator nerve is considerable. Very importent is correct intraoperative diagnosis of those complication and the knowlege of repair techics.

Methodology The aim of reconstruction of demaging obturator nerve is to restore the apropriate function. That's the motoric nerve responsible for adduction and walking. Subtile disfunction of a sensation are possible as well.

Result(s)* 54 years old women with clarocellular endometrial cancer with Massive infiltration of cervix, was admitted to Oncological Center for surgical treatment. She was previously hospitalized in Oncosurgery department due to isolating chanage in paraaortic lymphnodes. The enlarged nodous were removed. In $\mathrm{H}-\mathrm{P}$ the suspicious of the endometrial cencer as primary focus were given. In CT and MRI examinations the selective pathology of lymphnodes of the left pelvic side were detected. The radical hysterectomy type B with selective lymphadenectomy of the left side were done. During dissection of oburative space the iatrogenic damage of the obturative nerve were detected. The preparation of whole obturative nerve were performed end to end anastomosis with 6.0 monofilament suture was made. The surgery were continued due to qualification. In postoperative observation the patient didn't claimed any mobile disfunction of the left leg. In neurological examination discreet symptoms of disfunction could be observed, which subside in a few weeks.

Conclusion* Described case proves that laparoscopical technique has the benefits not only in minimally invasive treatment of endometrial cancer but in neural complications as well what could be much more difficult in traditional laparotomical route.

\section{ENDOMETRIAL CANCER IN PRE-MENOPAUSAL WOMEN: CLINICAL AND PROGNOSTIC FACTORS ANALYSIS IN A TERTIARY CENTRE}

R Bermejo Martínez*, MDLR Oliver, C Álvarez Conejo, G López González, B Gil Ibáñez, JM Seoane Ruiz, Á Tejerizo García. University Hospital October 12, Madrid, Spain

\subsection{6/ijgc-2021-ESGO.162}

Introduction/Background* Management of endometrial cancer (EC) in pre-menopausal women represents nowadays a clinical challenge. The median age of diagnosis of EC is 65 years. Prevalence has increased in the last years in younger people related to increased risk factors. Around $20 \%$ of cases are diagnosed before menopause. EC typically presents with abnormal uterine bleeding. The diagnostic evaluation of premenopausal patients with this main clinical feature supposes an additional difficulty. The aim of our study is to evaluate the clinical and pathologic factors associated with EC in this group.

Methodology Retrospective unicentric study of 244 patients with EC diagnosed, treated and followed-up at Hospital Universitario 12 de Octubre (Madrid, Spain) between 2013 and 2020. Exclusion criteria were neoadjuvant chemotherapy or primary radiation as treatment. Menopausal status at diagnosis was used to classify patients into two groups.

Result(s)* A total of 244 patients were included, of which 45 (18.4\%) were pre-menopausal and 199 (81.6\%) were postmenopausal. Demographic and pathologic characteristics of the two cohorts are described in table 1.

Conclusion* In our institution, premenopausal status was associated with a lower parity record $(1.1 \pm 0.8$ vs $2.15 \pm 1.3$, $\mathrm{p}=0.04)$, lower prevalence of hypertension $(13.0 \%$ vs $59.4 \%$, $\mathrm{p}=0.001)$, higher rate of smokers $(28.9 \%$ vs $14.9 \%, \mathrm{p}=0.039)$ and endometrioid histology $(97.8 \%$ vs $83.5 \%, \mathrm{p}=0.01)$. Hysterectomy was performed in all cases, and only one premenopausal patient underwent ovarian preservation. A total of $39.1 \%$ and $38.0 \%$ of the patients had pelvic lymph node assessment in the premenopausal and postmenopausal groups, respectively $(p=0.4)$ and paraaortic in $37.0 \%$ and $30.0 \%$ $(p=0.19)$. There was no significant difference in stage distribution, grade differentiation, myometrial invasion or lymphovascular space invasion, between the two groups. The rate of administration of adjuvant chemotherapy was $15.6 \%$ in premenopausal and $13.8 \%$ in postmenopausal patients $(\mathrm{p}=0.75)$ and $28.9 \%$ and $37.9 \%(p=0.26)$ of adjuvant radiotherapy, respectively. According to these results, in our population, there was no significant difference in the favourable clinical prognostic factors in premenopausal women. The retrospective nature of the study and the small size of the cohort could justify these findings. 